\title{
Re-energizing enquiry among our young professionals
}

\section{ChandraSekhar Roychoudhuri}

ChandraSekhar Roychoudhuri, "Re-energizing enquiry among our young professionals," Proc. SPIE 10452, 14th Conference on Education and Training in Optics and Photonics: ETOP 2017, 104520E (16 August 2017); doi: $10.1117 / 12.2266449$

SDIE Event: 14th Conference on Education and Training in Optics and Photonics, ETOP 2017, 2017, Hangzhou, China 


\title{
Re-energizing Enquiry among our young professionals ChandraSekhar Roychoudhuri ${ }^{\mathrm{a} *}$ \\ ${ }^{a}$ Department of physics, University of Connecticut, Storrs, CT, USA 06269-3046 \\ *Chandra.Roychoudhuri@uconn.edu
}

\begin{abstract}
Children are born with enquiring mindset. They keep on asking questions to explore, understand and take part in their environment. However, modern educational systems discourage persistent enquiring questions. Most students, graduating from college, can use their enquiring faculties only to solve problems at hand. They accept the theories taught as the final models for the laws of nature. They safely assume that no further deeper enquiry is needed. This is a disturbing collective tendency counter to our continuously evolving nature. We should also consciously train our minds to evolve continuously by persistently asking enquiring questions. Therefore, we suggest that we take pro-active steps to re-energize the enquiring mindset among our young professionals by organizing enquiry forums for students from all international Student Chapters during most of our optical society meetings. Panels of volunteer senior scientists should encourage deep enquiring questions from the students. In this paper, as examples, I will present a set of enquiring questions in the field of optics that we have been underscoring to students. This is one of the three papers by this author for this conference, ETOP 100-43. Since scientific content-wise they complement each other, the readers should consult the others. They are: ETP100-36, "Consequences of repeated discovery and benign neglect of non-interaction of waves" and ETP100-83, and "Demonstration and implications when 50\% beam combiners can behave as 0 or $100 \%$ reflector/transmitter inside some interferometers."

Keywords: Contradictions in classical and quantum optics; Creating safe platforms to raise enquiring questions; Energizing enquiry among students; Leveraging Student Chapters to promote enquiry; Use of all optical society meetings to "re-energize enquiry".
\end{abstract}

\section{INTRODUCTION}

\subsection{The Problem}

As a longtime industry engineer in USA and a professor in India, Mexico and USA, I have sensed that our advanced educational system effectively (not directly) discourages asking difficult and deeper enquiry driven questions in classes. Therefore, students get used to and become accustomed to accept the prevailing working theories as the final theories without further deliberations, and/or understanding the subtleties behind the relevant phenomena they are studying.

We know that all 1 to 2-year olds, all over the world, irrespective of economic classes or religious persuasions of their parents; barrage the adults around them with simple, as well as deeply enquiring, questions persistently. However, our educational systems, almost all over the world, eventually succeed in seriously dampening out these genetically inherited enquiring minds of the majority of our children world-wide. Therefore, nationwide school systems in many countries, at the lower levels, are now active in implementing enquiry driven education. ‘

How do we re-inculcate enquiring trend among those who have already graduated while indirectly trained not to ask difficult questions? These young professionals are already in the pipeline to lead our academic and industrial institutions. Should we not try to develop a forum to encourage them to re-develop their enquiring mindset so they naturally become more creative again?

\section{$1.2 \quad$ Objective and Approach}

The key objective is to create forums (conference workshops, papers and continue through web discussions) where our young scientists are encouraged and enticed to present their enquiring questions designed for deeper understanding of optics/physics phenomena that they have studied, or are studying. We can easily start with the members of all the international Student Chapters as question generators. Then we need to solicit experienced volunteers, out of industry scientists and professors, to help generate detailed responses to these questions. Selected enquiries that have deeper pedagogical values and/or connect/challenge some of the foundational postulates should be presented as conference 
papers and published as Educational Papers. The ETOP publication forum (run by ICO, SPIE \& OSA) can become the generic platform for publication of these papers. However, to engage the maximum number of international students, we need to organize separate "Enquiry Sessions" during all major conferences organized by OSA, SPIE, IEEE-Photonics, etc. We can also solicit donations for yearly awards to students and scientists generating the best "Enquiring Question" and the best "Scientific Response". This could be a long-term enticement to make the initiative, "Re-energizing Enquiry”, successful and long lasting.

\subsection{The specific content of this article}

Methodology of thinking behind scientific explorations and research is many million years old, which started with the human evolution as an enquiring species. For a deep history of the evolution of modern scientific thinking, the readers are encouraged to consult these references [1-8].

The phrase "evidence based science" has recently become popular in the press while some segments of our society are challenging the conclusions of the scientific observations; even biological evolution is being denied. In Section 2, I will briefly underscore the logical reason behind perpetual Scientific Revolutions [8]. Evidence is measured data. However, no amount of data can ever reveal all the details behind interactions that give rise to the data in our instruments, as has been discussed in section 2. This is why our knowledge perpetually remains incomplete. In other words, we must not declare any working theory as our final theory and the final knowledge of nature. We still really do not know what the electrons and photons are made of in spite of highly successful and advanced theory of quantum physics. This is the reason why we need to promote perpetual enquiring mindset among our young scientists.

Section 3 provides the core content of the article. I present a number of specific enquiring questions related to different optical phenomena, simply as examples to inspire enquiry. The purpose is to elucidate existing contradictions and logical in-congruencies within the working theories that we gloss over without underscoring. I hope that these examples will encourage our students to identify newer contradictions and logical in-congruencies, and hence, generate newer enquiring questions. This would lead to the development of the next generation better theories.

\section{WHAT IS MEASUREMENT? “EVIDENCE BASED SCIENCE” DOES NOT REPRESENT OUR FINAL KNOWLEDGE ABOUT NATURE!}

We have developed the assumption that we all think objectively while interpreting scientific observations and experimentally gathered data, as if no further discussions about the interpretations are necessary. In reality, we all have slightly different templates for logical thinking to create self-consistent meaning out of the same set of observations. Lack of explicit discussions in classes about this inherent divergences in our thinking have slowed down the continuous enhancement and evolution of our "correct" theories. I illustrate this point in the next two sub-sections.

\subsection{Two convergent views from two dramatically different ancient civilizations, West and East}

Some 6-thousand years old eastern allegorical story tells us that: We are all "blind" as far as our perfect objectivity is concerned (Fig.2.1, 1st image). Therefore, we must collaborate to discover the deeper truth. We know today that sustainable evolution in nature derives from collaboration between intra-species members and inter-species. The individual model of the Cosmic Elephant derived by individual sensorial inputs from five blind men (Fig.2.1, $1^{\text {st }}$ image) is quite limited. They perceived the elephant as a snake, or a pair of spears, or a pair of fans, etc. (Fig.2.1, $2^{\text {nd }}$ image). However, once they trusted each other to collaborate and try to synthesize a living animal, their model converged to a reasonably close model of an elephant, albeit not the complete and perfect reality. Our working knowledge of science has also been evolving in a similar way with the contributions from innumerable scientists working through millennia. Separate individual theories, validated by experimental data, are somewhat similar to modeling the Cosmic Elephant! However, collaborative synthesis will always bring out continuously enhanced reality[5]. 

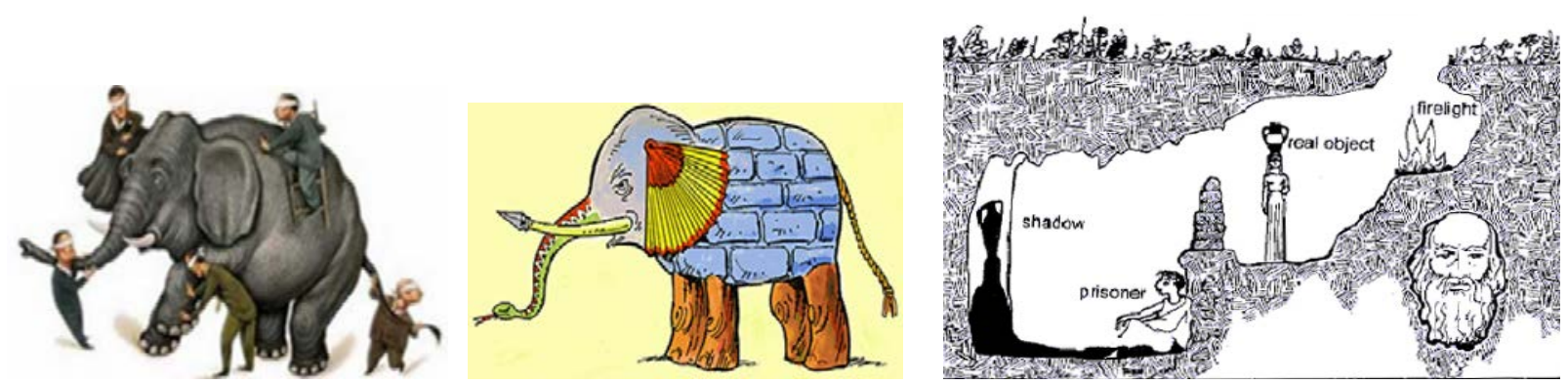

Figure 2.1 Left Two Elephant Images: The allegorical story tells us that five blind men, individually, perceived the elephant as a snake, a pair of spears, or a pair of fans, etc. However, once they trusted each other to collaborate and try to synthesize a living animal, their model came reasonably close to the reality, albeit not the complete reality. Extreme Right Image: Plato ( 424 - 348 BCE) warned people that our observations are only "shadows" of interactions that goes on between interactants in our instruments. He gave the analogy of the limitations of drawing conclusions about the outside world based on the shadows of the outside objects cast on a cave-wall, by people who were imprisoned inside the cave permanently. [Figures have been collected from Google Images]

2.2 Philosophers' “Measurement Problem” is, in reality, the "Information Retrieval Problem"

Let us dissect the key and essential steps behind any data gathering measurement [Ch.12 in ref.9]

(i) Measurables Are Transformations: A properly designed instrument can register the desired data only when the detector undergoes some measurable physical transformations.

(ii) Preceded by Energy Exchange: However, there cannot be any physical transformations without some energy exchange.

(iii) Guided by Forces of Interaction: The desired physical transformation(s) through some energy exchange has to be guided by an allowed force of interaction.

(iv) Must Experience Physical Superposition: Interactants must be within the physical range of each other's force of influence to be able to interact and exchange energy; and then undergo physical transformations. Thus, all interactions producing transformations must be range-defined "local"; because, all the known four forces have finite spatial range to be effective. An electron and a proton, separated by distance of one meter, cannot bind themselves to form a Hydrogen atom.

(v) Through Some Physical Interaction Process: Energy exchange between interactants in an instrument, to give rise to some measurable data, must be preceded by some physical transformational interaction process under the guidance of the allowed force between them. These interaction processes are, almost never, directly visible to us. The attempts to understand \& visualize these invisible interaction processes will anchor us to advance our understanding towards real cosmic logics (reality).

(vi) The Information Retrieval Problem: Thus, experimental data, validating a mathematical theory, cannot provide us with all the necessary information directly relevant to the interaction processes that give rise to the data. Only our imaginations to visualize the interaction process can help us advance; or, invent newer instrumental tools that are capable of creating images of such interaction processes. Mathematical theorems, however elegant, cannot decisively retrieve all the unknown information. It can provide some guidance to construct logically congruent postulates as what are the detailed steps (images) behind the interaction process. However, these postulates will still be our conjectures, not the cosmic truth. This is why we must teach the students to keep on challenging the foundational postulates behind all working theories and assure consistent evolution in scientific theories.

We want to underscore the following point. The evidence-based science is the best approach, which we have been following for millennia; and yet these data-validated-theories cannot give us the complete objective picture behind the interaction processes modeled by the theory. Hence, we should not consider data-validated-theories to represent our final knowledge about nature. We need to inculcate this mode of thinking among our young scientists so their enquiring minds always remain vigilant against accepting any working theory as our final knowledge of nature. 
The following two quotations from two major physicists of modern human history will further strengthen my points. Newton supposed to have said, "If I have seen further than other men, it is by standing on the shoulders of giants". Since Newton's time we have now accumulated thousands of more accomplished scientists. We must respectfully learn to stand on the top of the pyramid built out of the great minds of these scientists to increase our knowledge horizon. We must not bow down our heads out of our cultural "messiah complex" to these scientists and, thereby, reduce the growth of our knowledge horizon.

Einstein, a short time before his death, was supposed to have said, “.... After 50 years’ of brooding over the question of what are light quanta; I still do not understand it!” This is the sign of a truly honest enquiring mind of a scientist. Einstein's "indivisible light quanta" ("photon”) has got him the Nobel Prize and has been the prevailing mode of thinking by physicists until today; and yet, Einstein was questioning his "successful” postulate because of many built-in contradictions he was recognizing. In fact, it is this enquiring view of Einstein that has inspired the author to continue to challenge the postulate of "indivisible light quanta"!

\section{EXAMPLES OF ENQUIRING QUESTIONS IN OPTICAL SCIENCES}

Framing the questions determine the answers. Therefore, framing new questions will lead us to explore and discover new knowledge. That has been the history of evolution of human knowledge. History also tells us that all working theories eventually yield to newer and better postulates [8]. Therefore, research students should be encouraged to seek out proactively the prevailing contradictions and logical incongruences that are hiding in current working theories and their foundational postulates. Because, all earlier postulates, however rational they appeared to be during their earlier times, were formulated based upon insufficient knowledge of the deeply interconnected universe. There is always room for new research, develop new knowledge in every field.

\subsection{Diffraction Phenomenon. [Non-Interaction of Waves (NIW) built into it.]}

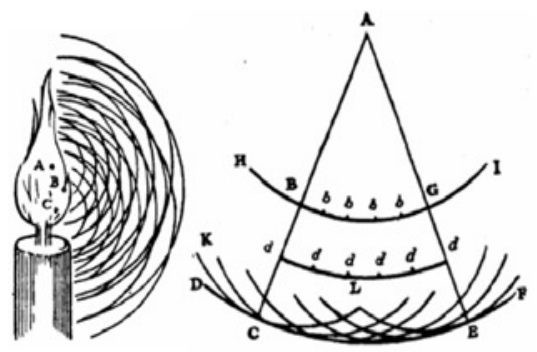

Figure 3.1.1 Diagrams sketched by Christian Huygens in his book [10] explaining the intrinsic properties required by waves to keep on propagating perpetually energized by cosmic ether as a tension field. Each point on every wave front functions as a source for a secondary wavelet. He also explicitly stated that his secondary wavelets keep on expanding through each other without influencing (interacting with) each other. This is Non-Interaction of Waves (NIW).

(i) Why do we ignore Huygens principle of Non-Interaction of optical waves, while we all use his key postulate of secondary wavelets as is evidenced through our use of Huygens-Fresnel diffraction integral?

Huygens explicitly mentioned in his book [10] that his secondary wavelets propagate unperturbed through each other, which is evident from Fig.3.1.1 copied from his book.

(ii) What is the source of energy that keeps on generating the secondary wavelets during the entire cosmic journey of light waves emitted by a star in the most distant galaxy?

Huygens and Newton were familiar with the need for a tension field (ether) that can support the perpetual propagation of light waves across the cosmic space without any "sustained push" from the emitting source. (Formulation of Doppler Effect, the effect of source movement, was developed much later, in 1842.). Acceptance of space as a Complex Tension Field (CTF) holding all the cosmic energy is in the process of enhancing future of physics significantly [See Ch.10-12 in ref.9].

Why do we use non-causal Fourier component frequencies to compute pulse broadening in propagation instead of directly using diffraction theory? 


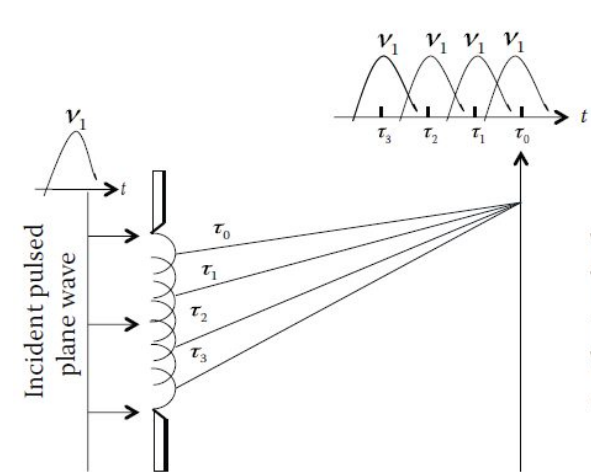

(a) Single frequency pulse

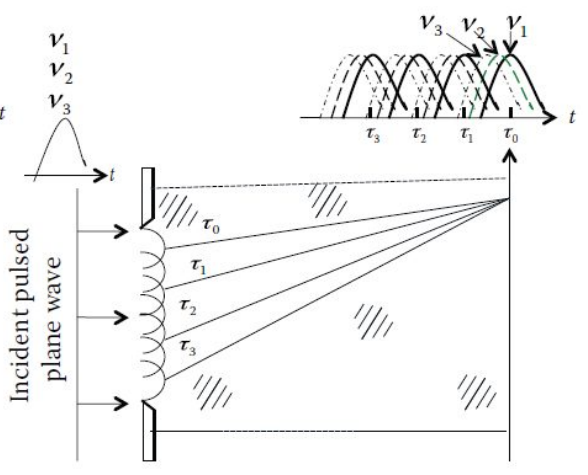

(b) Multi-frequency pulse

Figure 3.1.2 Proposed pictorial model to compute diffractive pulse broadening using Huygens Principle, both for free space (left diagram) and for dispersive materials (right diagram), when the pulse physically contains multiple optical frequencies. Each light pulse should be propagated taking its carrier frequency as the real physical frequency [see Fig 4.6 in 9].

Huygens Principle clearly implies that the physical length of a short pulse would appear from un-broadened response at on-axis location to steadily broadening at various off-axis locations, Fig.3.1.2b. This obvious physical property of Huygens principle is still not explicitly recognized in physical optics. This has new engineering implications in computing pulse broadening due to material "dispersion" in fiber optics communications [See section 4.5 in ref. 9].

(iv) Etc., etc.

\subsection{Spectrometry Theory (see Ch.5 in ref.9)}

In classical spectrometry, we derive the spectrometer response function using the non-causal continuous wave (CW) that does not exist in nature. At least, we recognize that this CW response function of finite width does not represent the existence of real physical frequencies. Therefore, we de-convolve this CW response function from the data for "CW" light to derive the actual physical spectrum.

(i) Then, why do we assume that the broadened fringe generated by a pulsed light is due to the physical presence of Fourier frequencies?

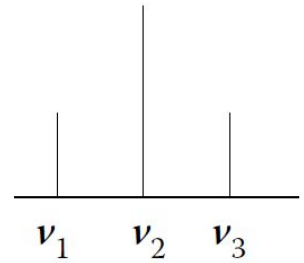

3-mode laser

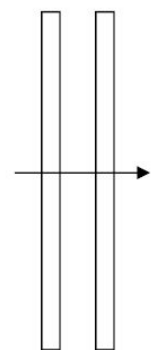

Grating or FP

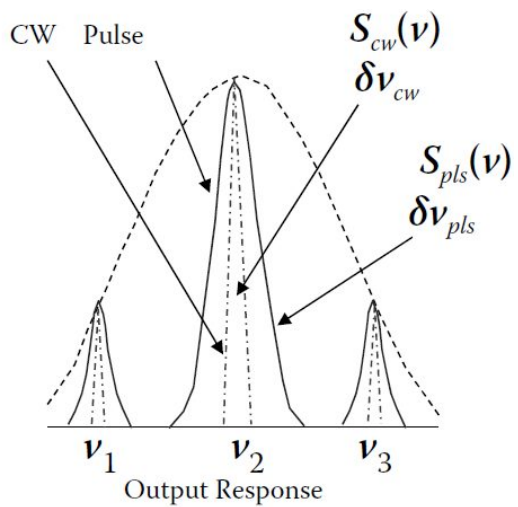

Figure 3.2. The output response of a spectrometer shows fringes of finite width (dashed vertical curves on the right diagram) even when the input signal is infinitely narrow thee CW laser modes (shown on the left). We correctly recognize this width as the CW instrumental response function. The output response would be wider (solid vertical curves) if we allow a simple screen to chop out a single pulse from the 3-mode CW laser. We should recognize this widening as the pulse response function of the spectrometer, rather assuming that the width is due to existence of Fourier frequencies in the chopped pulse [see Fig. 1.1 in ref.9].

(ii) What are the physical reasons behind accepting that a pulsed light is always built out of the summation of complex amplitudes given by the mathematical Fourier transform of the amplitude envelope of the pulsed light? Are there any supporting postulates? 
Notice that our fundamental diffraction theory, representing light propagation through any linear medium, is built upon non-interaction (non-interference) of wave amplitudes.

(iii) Can spectrometers, consisting of linearly and instantaneously responding optical components like, grating or pairs of beam-splitters (Fabry-Perot's) can literally execute the Fourier transform algorithm?

The required steps behind Fourier algorithm are quite complex. First, the shape of the envelope function of the pulse travelling at the speed $c$ needs to be registered and stored in an accessible memory bank. Then the complex Fourier integral operation has to be executed over the complete envelope to determine the Fourier frequencies. Then the instrument has to respond to all the frequencies individually to generate the individual frequency response function. Then all these individual amplitude response functions have to be summed before delivering to the external detector.

(iv) Are these steps causally executable by a passive and linear media (instrument)? Alternatively, do we need to develop a causal theory of spectrometry?

(v) Why do we keep using, mathematically correct, but physically non-causal Fourier monochromatic waves when it represents a non-causal signal existing in all space and all time?

Such a signal violates the law of conservation of energy.

(vi) Why do we get right measurable result most of the time predicted by non-causal Fourier theorem most of the times (not always)?

Usually, the validity appears when the spectral recording is carried out by long time integration.

(vii) Etc., etc.

\subsection{Coherence Theory}

Degradation of fringe visibility steadily increases in both the cases for white light and pulsed light interferometry as the relative path delay is increased. However, the physical reasons behind these two phenomena are distinctly different.
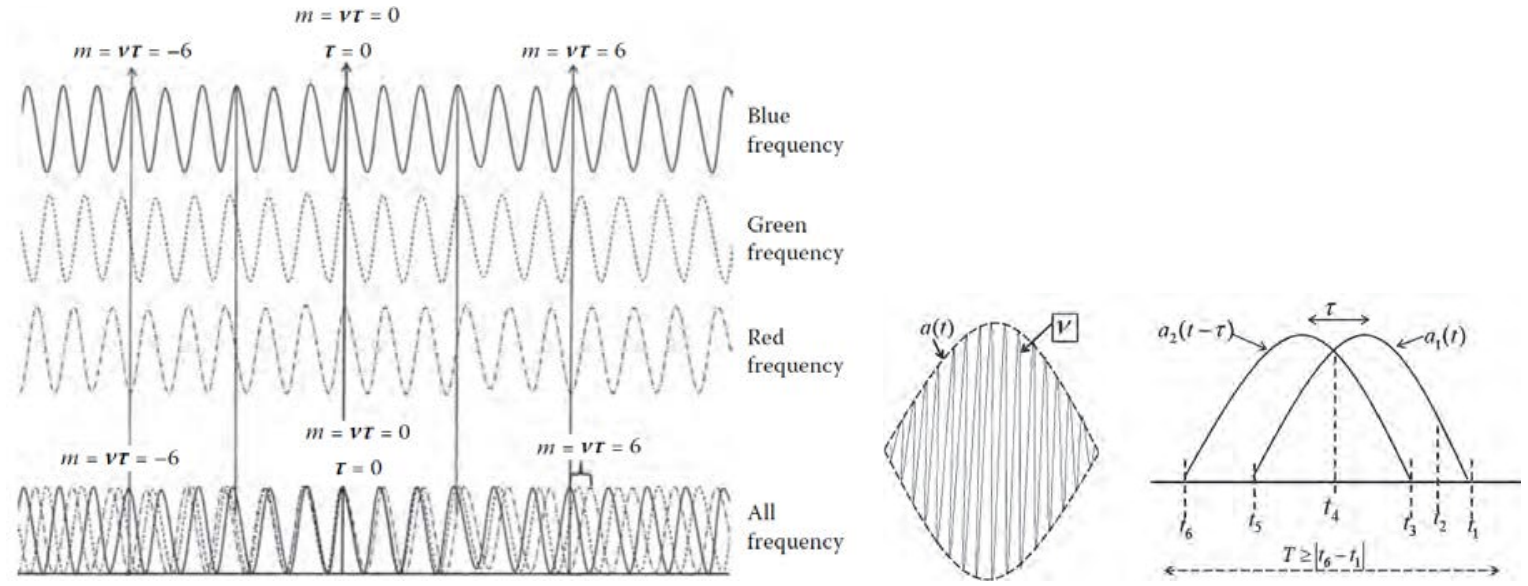

Figure 3.3. Left Diagram (see Fig.1.2 in ref.9): Some of the most precise measurements using optical interferometry is done by using broadband or white light. Highly "incoherent" white light can produce quantifiable fringes for measurements for small path differences (see the very bottom sketch above). The fringes wash out for increasing relative path differences. This can be appreciated from the bottom of the left figure. The red, green, and blue fringes have different spatial frequencies. When they all are present simultaneously, the fringes show increasing wash-out effect as one move further from the "zero-path-delay' position. Right Diagram [see Fig.6.1 and 6.2 in ref.9]: In pulse autocorrelation experiments, a single pulse is replicated and superposed with different relative delays. Replicated pulses are phase steady; not incoherent. Fringe visibility degrades because unequal amplitudes are superpose, not due to physical presence of Fourier frequencies.

(i) Why do you we define degradation of fringe visibility (less than unity for the auto correlation function) as due to "incoherence" of light when we use white or multi-frequency light?

See Fig.3, left diagram. Starting from Michelson in late 1800 and till today, we have been carrying out extremely precise displacement measurements using white light (broad frequency band). Non-Interaction of Waves, or NIW, already defined in Section 2.1, clearly implies that each separate frequency wave-pulse of white light is completely independent of each other. When each wave packet is replicated by an interferometer, the pair will stimulate a 
detector with phase-delayed, but phase-steady signals. However, the spatial frequencies of the fringes for each wave group will vary in space (relative delay) with the frequency (wavelength) of the specific component of light. Close to "zero" path delay location, fringes due to different frequencies remain spatially coincident and hence gives excellent fringe visibility. Further away from the "zero" path delay, the aggregate fringes will tend to wash out the discernibility of fringe-visibility. These observations imply that the degradation of fringe visibility due to the presence of multiple frequency should not be defined as "degradation of coherence"; or, "white light is incoherent".

(ii) Why do you we define degradation of fringe visibility (less than unity for the auto correlation function) as due to the presence of Fourier frequencies, when we use a pulsed light in the interferometer?

The real degradation of fringe visibility arises due to superposition of unequal amplitudes of the two replicated but displaced pulses with steady phase relation. See Fig.3.3 right diagram. In the last section, we have already explained that there is no postulate supporting the existence non-causal Fourier frequency in a pulsed light. Even when multifrequency phase-steady light beams are summed or superposed (Fourier transform theorem), the waves cannot generate a new and re-grouped light pulse by virtue of the NIW property.

(iii) Etc., etc.

\subsection{Mode Lock Theory}

Basic texts on laser mode-lock theory simple sums, as per Fourier theorem, the periodic set of longitudinal modes allowed by a laser cavity under the mutually steady phase condition. It obtains ( $\sin / \sin )$ amplitude envelope with mean central carrier frequency as in Eq.1 (see also the right sketch in Fig.3.4). In reality, mode locked lasers clearly show the existence of the independent "comb frequencies"; which have been finding wide variety of engineering applications, including the most precise atomic clocks.

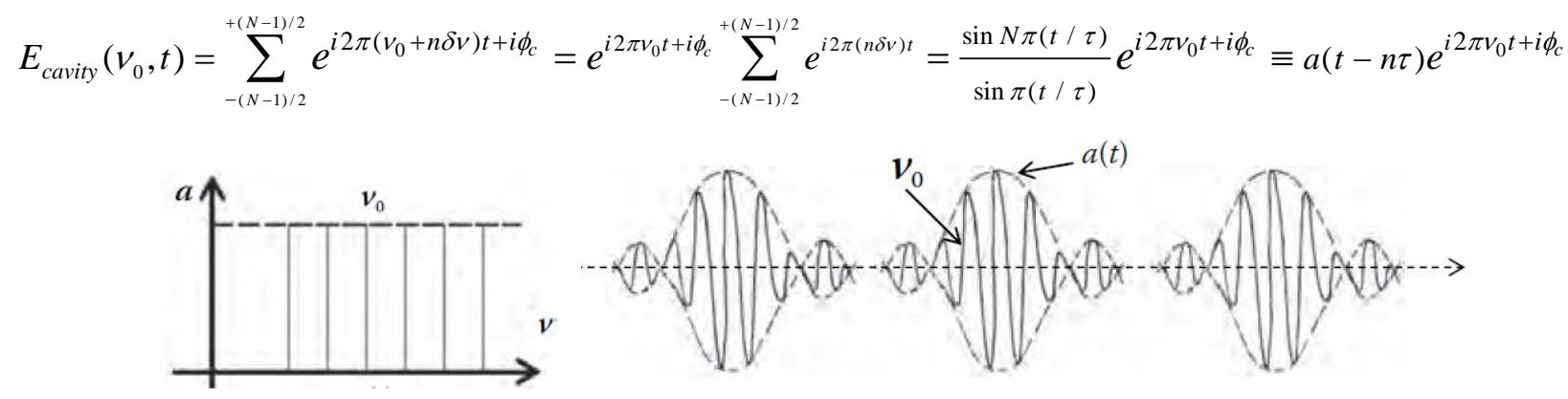

Figure 3.4. Mode lock theory in basic textbooks sums the amplitudes of a periodic array of phase-steady frequencies (left diagram) to obtain a continuous train "mode locked" pulses. Clear implication is that wave amplitudes are interacting and re-grouping their energies, which violates the NIW property of light, as explained earlier. In reality, it is the time-periodic intensity response function of the intra-cavity mode-locking device, which functions as a time gating device, enforcing the intra-cavity gain of a train of reverberating light pulses. [see Fig.7.1 in ref.9]

(i) Why do we keep summing wave amplitudes to derive the generation of light pulses out of mode-locked lasers violating the generic NIW property of waves?

(ii) Why do we keep presenting the above mode locking theory even though no measured pulse shape ever corroborate the $\sin ^{2} \mathrm{Nx} / \sin ^{2} \mathrm{x}$ model? In reality, they vary from system to system as Lorentzian, Gaussian, etc.

(iii) Why do we keep presenting the above erroneous mode lock theory even though it predicts that the pulse train contains only a single mean Fourier frequency, when experimentally we always find a set of oscillating "comb frequencies" that are being used for wide range of engineering applications, including constructing the most precise atomic clocks?

(iv) Etc., etc. 


\subsection{Dispersion Phenomenon (frequency dependent light velocity)}

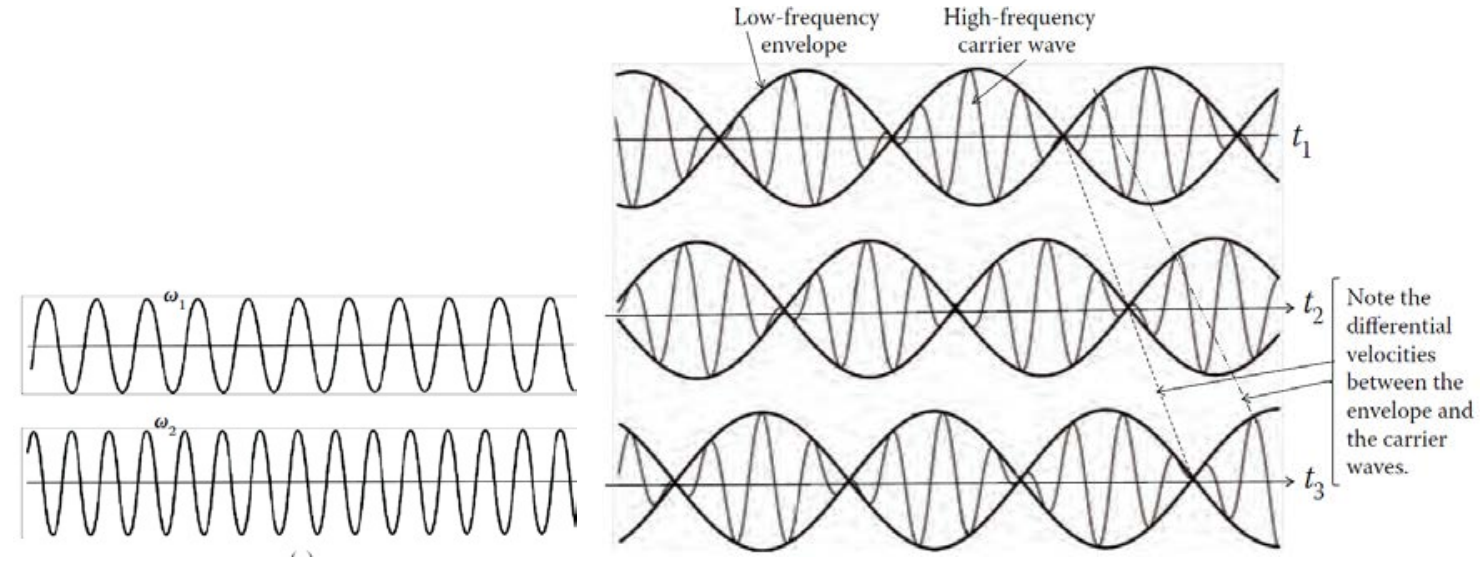

Figure 3.5. Left Diagram: Two phase steady continuous waves. Right Diagram: Mathematical summation of two phase-steady continuous cosine waves for two different carrier frequencies will give a mathematical wave with a new carrier frequency of value equaling the mean of the sum frequencies modulated by an envelope wave, which is the mean of the difference frequency. Since materials impose frequency dependent velocities, one mathematically finds that the envelope wave travels with a group velocity that is different from the phase velocity of the resultant carrier wave. This is the standard textbook theory. However, it violates the NIW property of waves, underscored earlier. The two component waves will co-propagate unperturbed by each other, as long as the medium supporting the waves remain liner and non-interacting with the waves [see Fig.8.4 in ref.9].

(i) Why do we keep on teaching the dispersion theory using a wrong basic postulate that waves amplitudes interact to re-group themselves?

Reference 9 gives a number of experiments where different optical frequencies are superposed to demonstrate that the amplitudes do not physically sum. However, when they simultaneously interact with a detector, the observed Superposition Effect gets determined by the width of it frequency response and time response functions. For example, $\mathrm{Rb}$-gas atoms, having very sharp quantum transition levels, cannot respond to the mean frequency, even when the copropagating two-phase steady optical frequencies are symmetrically straddling above and below the atomic energy level [11].

(ii) When one couples in a light pulse with a single carrier frequency into a single mode fiber, would the pulse propagate with the group velocity or the phase velocity dictated by the carrier frequency?

(iii) Is the data pulse broadening, routinely measured in long-haul single mode fiber, really due to component Fourier frequencies of the individual data pulse envelope?

(iv) Why do not the communication engineers use the Fourier transform of the entire train of data pulse to compute the pulse broadening, even though a long segment of the pulse train is mutually phase steady ("coherent")?

(i) Should the pulse broadening in single mode communication fiber be re-derived by employing diffractive propagation theory instead of Fourier frequency-dispersion theory? (See also section 2.1 and Fig.2.1.2).

(ii) Etc., etc.

\subsection{Polarization Phenomenon}

We are taught that orthogonally polarized light beams, even if they are phase-steady, do not interfere with each other. In light of NIW property, this is correct; no light beams interfere in the absence of interacting materials. Therefore, the above statement is superfluous. However, when we insert a Polaroid in front of the detector array (see Fig3.6.1) precisely bisecting the $90^{\circ}$ orientation of the two polarized beams; we could register perfect visibility fringes (the right photograph in Fig.3.6.1). Obviously, the interaction physics is in the detector. 

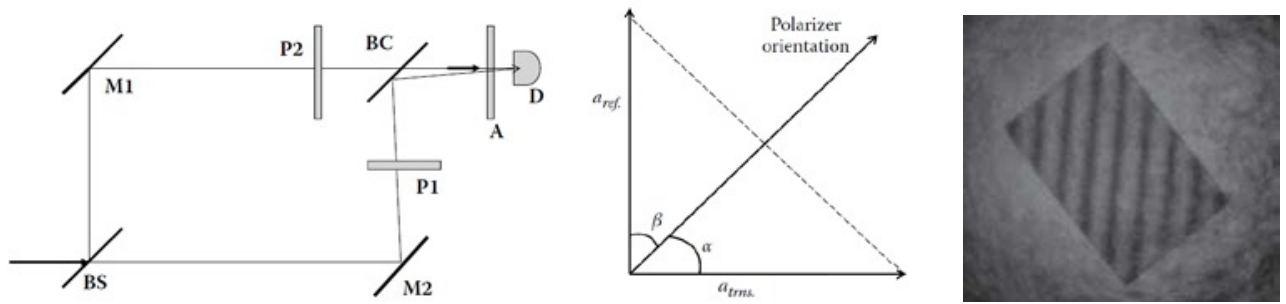

Figure 3.6.1 Left Diagram: Two orthogonally polarized beams were generated in the two arms of a Mach-Zehnder interferometer (MZI) and were superposed non-collinearly on the beam-combiner (BC). (The MZI is in fringe mode when only a detector array can register spatial fringe intensity variation.) However, with orthogonally polarized beams, it does not produce any fringes until a polarizer is inserted bisecting the two orthogonal direction of polarizations. Right Diagram: The recorded fringes visible only within the boundary of the Polaroid. No fringes were visible outside boundary of the Polaroid (see Fig.1.3 in ref.9).

(i) Why do we define that only orthogonally polarized light beams do not interfere; when generically, no light beams interfere?

(ii) Why do we ignore, while explaining the interference of light that the obvious physics of excitability of detecting molecules, as uniaxial diploe, is at the core of registering detectable signal?

In photographic plates, the measurable data appears due light induced breakdown of Ag-Halide molecules (after chemical development). For modern photo detectors, it is the release of electron after the molecular dipole complexes release detectable electrons. In both cases, the electric vector of light beam induces uniaxial dipolar stimulation in the detecting molecules. A molecule, in an isotropic medium, can become a dipolar oscillator in any direction dictated by the E-vector. When multiple parallel and in-phase E-vectors stimulate the same dipole complex, it executes the sum total stimulations and absorbs energy proportional to the square modulus of the sum of all the E-vector amplitudes. However, when two equally strong E-vectors try to stimulate the same dipole in orthogonal directions, it cannot execute effective summed oscillation and hence cannot absorb energy to display superposition effect.

(iii) Can the molecules of a Polaroid facilitate Superposition Effect in an MZI set for collinear superposition?
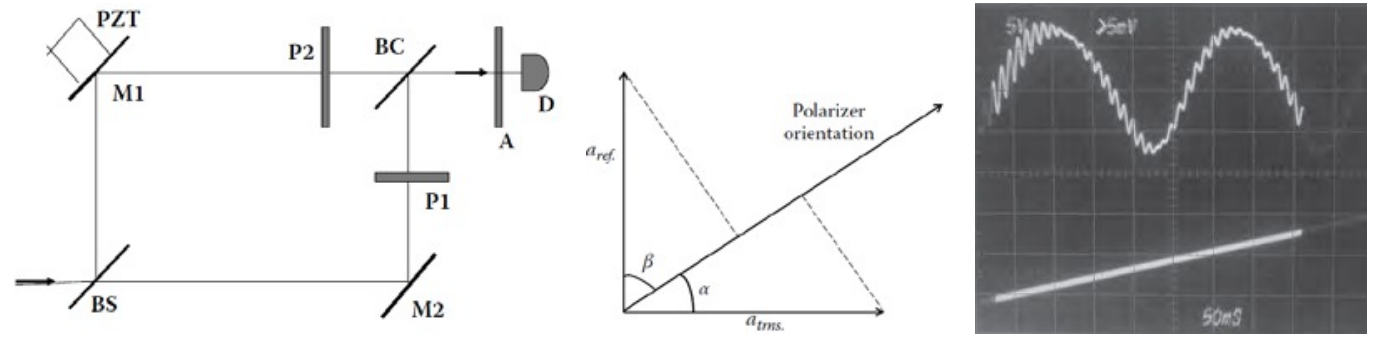

Figure 3.6.2 An MZI in collinear superposition mode for a pair of phase-steady orthogonally polarized light beams. The detector can observe the superposition effect only when one of the mirrors is scanned to introduce relative phase delay between the two beams along with a polarizer before the detector. The two superposed beams on the detector are now parallel, dictated by the polarizer. The detectors now can respond to the superposition effect. The beam combiner (BC) remains passive to the orthogonally polarized beams as in the case for non-collinear superposition above (Fig.3.6.1).

When an MZI is set in collinear superposition mode, the relative phase variations between the two beams have to be generated by scanning one of the mirrors. The polymer molecules in a Polaroid are perfectly stretched and aligned in one particular direction of "polarization". No matter which polarized light is incident on them, they will take the $\cos \theta$ amplitude projections of all the different E-vectors and transmit them as collinear, non-interacting beams (NIW). A Polaroid cannot execute the square modulus operation. The detector behind the Polaroid takes the square modulus of the two collinear beams and absorbs energy accordingly; which appears as scanned fringes on an oscilloscope (the right photo in Fig. 3.6.2).

\subsection{Can "indivisible single photon" generate interference effect?}

See the paper in this volume \#ETP100-83, "Demonstration and implications when $50 \%$ beam combiners can behave as 0 or $100 \%$ reflector/transmitter inside some interferometers" [12]. In these set of experiments, we demonstrate that a classical MZI in scanning mode, as shown in the above Fig.3.6.2, the beam combiner can re-direct all the energy of both 
the incident beams into one or the other direction, depending upon the relative phase delay between the two incident beams from the opposite directions. This is a classically predicted result. The aggregate molecules of the dielectric boundary layer of the beam combiner are actively participating in determining how much energy from which beam goes in which direction. No photo-active quantum material is necessary. However, the key point to recognize is that both the light beams from the opposite directions must be present simultaneously to generate this simple classical superposition effect. Then, the question is this:

(i) How can a single indivisible photon in the interferometer can bring to bear two different phase parametric values from the two opposite sides of the beam combiner at the same instant?

In another paper in this volume, ETP100-36, "Consequences of repeated discovery and benign neglect of non-interaction of waves" (13), we show historical, experimental and mathematical evidences to establish that wave amplitudes do not interact (interfere) by themselves even when they are superposed.

(ii) How is it possible that a single photon, by itself, can generate superposition effect, when light waves do not interfere at all?

(iii) Should we then replace Einstein's “indivisible quanta” by Planck's divisible classical wave packet, while accepting the reality that binding energies of all photoelectrons are quantized in all materials: Our instruments can register only "clicks" because released photo electrons are discrete?

(iv) Should we replace Dirac's statement "A photon interferes only with itself" by "A detector's simultaneous stimulations due to multiple excitations engender superposition effect?" Frequency resonant detectors are at the root of engendering superposition effects, whether classical or quantum.

(v) Should we replace Dirac's photon as an "infinite Fourier mode of the vacuum" by "classical time-finite wavepacket mode of the vacuum" enforced on the cosmic complex tension field by excited electrical atomic and molecular dipoles?

(vi) How can "indivisible photons" be entangled when there are no force of interaction between light waves (or photons) and they obey the NIW property?

\section{EXTENDING HUYGENS’ ETHER CONCEPT TO MODERN PHYSICS}

People realized from old times that it is the surface tension field of water surface that facilitate perpetual propagation of water waves from the point of generation (say, where one gently and vertically drops a stone) to far distances until it dies out due to various dissipative forces. The same is also true for sound waves; it is the three dimensional pressure tension field of air. Therefore, ancient physicists were aware of the necessity of a complex tension field that fills the entire cosmic volume, which facilitates the propagation of light with a constant velocity across the entire galaxy.

The field of experimental and theoretical optical physics and the precision optical measurement tools have been at the foundation of promoting the evolution of most of the branches of sciences. This is true from ancient to modern times. Therefore, for the sake of inspiring all optical physics students, we are adding more enquiring questions by extending the concept that space is a stationary complex tension field [Ch.10 \& 11 in ref.9]. Therefore, for the sake of completeness, some more enquiring questions are presented here very briefly without extended explanations. These are more challenging enquiring questions. Some relevant papers can be down loaded from the author's website [14].

\subsection{Quantum and Relativity Physics}

I have already mentioned a number of the quantum optics related enquiring questions in the last section 3.7. Here are a few more questions beyond "photons".

(i) Should one contemplate replacing Born's interpretation of $\psi$ as an abstract "mathematical probability amplitude" by "real physical undulatory stimulation of internal structure of particles"?

If $\psi * \psi$ generates real numbers corresponding to particles real behavior, then $\psi$ must contain its real physical properties in the form of some real oscillatory amplitude characteristics rather than some abstract "mathematical probability amplitude" only. The square modulus of the complex Psi-function models the brief time averaging process when the resonance is identified before energy exchange through quantum transition. 

proportional to their kinetic energy"?

A principle of nature should not diverge under realistic conditions. De Broglie relation diverges as the speed of a particle tends to zero: $\lambda=\mathrm{h} / \mathrm{p} \rightarrow \infty$ as $\mathrm{v} \rightarrow 0$. If we assume $E_{k i n}=h f=(1 / 2) \mathrm{mv}^{2}$, where $f$ is the internal frequency of oscillation; then we can see that when $\mathrm{v}$ tends to zero, $f$ also tends to zero, along with the kinetic energy; without diverging like Broglie relation does. See section 2.11 in ref.13.

(iii) Is Bell's "inequality theorem" the correct logical guide to challenge the completeness of QM formalism? Can experimental violations assure the "completeness of QM" formalism?

QM accepts that the mathematical Principle of Superposition Principle (SP), the sum of amplitudes $\sum_{n} \psi_{n}$ is not directly observable. Because the sum of amplitudes does not facilitate any energy exchange between the waves to generate observable data in the absence of interacting materials (detectors, etc.). This also accommodates Huygens' claim of NonInteraction of Waves (NIW). Bell's Theorem mathematically models SP, and not the observable Superposition Effect (SE) that a detector generates after carrying out the square modulus operation and energy exchange. This promotes the acceptance of a non-causal concept of nonlocality in SE without having any foundation in the physical interaction processes (see section 2.2 above). Accept "Entanglement" only conditionally. All physical interactions are local in the sense that the interactants must be within each other's physical sphere of force field. Particles cannot remain entangled beyond the range of the QM allowed force of interaction.

Should we replace Heisenberg's "uncertainty principle” with "information retrieval problem”, as discussed in section 2.2?

It is not a principle of nature. It is an excellent mathematical theorem [15]. It is the human limitation of extracting all possible information about any natural entity, which generates indeterminacy, as we have discussed in section 2.2 above.

Should we replace Einstein's "relativistic Doppler effect" by the original "classical Doppler effect", where the two different Doppler shifts, due to source velocity and detector velocity are clearly discernible?

The Doppler shift suffered by a wave packet as it emerges out of a moving source is real, and the shifted frequency persists as a permanent physical change as it propagates through the stationary ether field. However, different moving sensors will perceive this same wave packet as having different carrier frequencies that are "apparent". Consistent success of the QM rules behind spontaneous and stimulated emissions require this proposed modification [16].

(vi) Should we contemplate replacing "4-D space" by "3-D space"?

We measure the 3D space with physical measuring "stick". We can "dilate" or "contract" such physical scales by changing the temperature or some other relevant physical parameters around the "stick". In contrast, we have no physical object that can directly measure the running time $t$. In the real world, we can only measure (count) the frequency of oscillations of diverse oscillators like household pendulum clock, or the laser light frequency for atomic clock, etc. We invert the frequency to derive a secondary parameter, the period or the time interval. Then by counting larger and larger number of oscillations, we derive the human concept of running time. We can also "dilate" or "contract" the frequency of oscillators using suitable change in the physical environment around them. Key primary mathematical parameters should be directly measurable as a physical property of something real in nature to keep our theories connected to nature's objective reality.

\section{CONCLUSIONS}

We have proposed a good number of enquiring questions for the beginning and advanced research students along with brief rationales and some references. We hope that this will generate serious participation by research student and scientists to develop a sustained environment that will keep on encouraging our students to keep on questioning the foundational postulates behind all working theories. This is the only way that we can avoid waiting for accidental appearance of another Newton, or another Einstein. We should not allow such uncertainty to continue! We should be proactive to encourage persistent enquiry and create the necessary secure environment. In the current scientific enterprise, such a culture does not exist now. We will have to create it. But, how? 


\section{REFERENCES}

[1] Wikipedia, “Scientific Method”, https://en.wikipedia.org/wiki/Scientific_method, (2017).

[2] Wikipedia, "Research”, https://en.wikipedia.org/wiki/Research (2017).

[3] Frank Wilczek, [A beautiful Question: Finding Nature’s Deep Design], Penguin Press (2016).

[4] Edward R. Dougherty, [The Evolution of Scientific Knowledge: From Certainty to Uncertainty], SPIE Press (2016).

[5] Nancy J. Cooke and Margaret L. Hilton, Editors; [Enhancing the Effectiveness of Team Science], National Academies Press (2016).

[6] Steven Weinberg, [To Explain the World: The Discovery of Modern Science], HarperCollins Publishers (2015).

[7] Mathias Frisch, [Causal Reasoning in Physics], Cambridge University Press (2014).

[8] Thomas Kuhn, [Structure of Scientific Revolution], 50th Anniversary Edition, Chicago University Press (2012).

[9] C. Roychoudhuri, [Causal Physics: Photon Model by Non-Interaction of Waves], Taylor and Francis, Hardcover, 2014, Paperback (2017).

[10] C. Huygens, Traité de la Lumière (drafted in 1678; but published in Leyden by Van der Aa, 1690), translated by S. P. Thompson as [Treatise on Light], London, Macmillan, 1912; See http://www.gutenberg.org/ebooks/14725 for free download from Project Gutenberg edition (2005). C. Huygens (1690), translated by Silvanus P. Thompson (1912), Treatise on Light, London: Macmillan, 1912; Project Gutenberg edition (2005).

[11] Dong-Ik Lee and C. Roychoudhuri, "Measuring properties of superposed light beams carrying different frequencies”, Optics Express, 11 (8), 944 (2003).

[12] C. Roychoudhuri, ETP100-83, "Demonstration and implications when 50\% beam combiners can behave as 0 or 100\% reflector/transmitter inside some interferometers”; SPIE Proc. ETOP (2017).

[13] C. Roychoudhuri, ETP100-36, "Consequences of repeated discovery and benign neglect of non-interaction of waves”, SPIE Proc. ETOP (2017).

[14] C. Roychoudhuri, Accessible copies of papers by the author from the web: http://www.natureoflight.org/CP/ (2017).

[15] C. Roychoudhuri, “’'Heisenberg's Microscope - A Misleading Illustration”, Foundations of Physics, Vol. 8, Nos. 11-12, (1978).

[16] C. Roychoudhuri, “Tribute to H. John Caulfield Hijacking of the 'Holographic Principle' by cosmologists”, Proc. SPIE 8833-15 (2013). 